\title{
Cuestionario de frecuencia de consumo de alimentos para estimación de ingestión de folato en México
}

\author{
Marcia Galván-Portillo, DSP,(I) Luisa Torres-Sánchez, DSP,(I) Raúl Ulises Hernández-Ramírez, MSc, ${ }^{(I)}$ \\ Miriam Aracely Anaya-Loyola, DSP.(2)
}

\author{
Galván-Portillo M,Torres-Sánchez L, \\ Hernández-Ramírez RU,Anaya-Loyola, MA. \\ Cuestionario de frecuencia de consumo de alimentos \\ para estimación de ingestión de folato en México. \\ Salud Publica Mex 20I I; 53:237-246.
}

\section{Resumen}

Objetivo. Evaluar la reproducibilidad y validez de la ingestión de folato (IF), estimada a partir de un cuestionario de frecuencia de consumo (CFC), considerando el factor de retención (FR) correspondiente al método de preparación empleado. Material y métodos. Al inicio del estudio se entrevistó a 497 adultos sanos; un año después se realizó una segunda entrevista a una muestra de 100 individuos seleccionados aleatoriamente, que no hubieran modificado su dieta durante el año previo; adicionalmente, para evaluar la validez de la IF, mediante radioinmunoensayo, se determinaron los niveles de folato sérico (FS). Resultados. La reproducibilidad de la IF fue de 0.36 y 0.34 para el folato sin y con FR, respectivamente. Se observó una correlación significativa entre la IF y el FS de 0.18 con FR y 0.21 sin FR ( $p<0.05$, respectivamente). Conclusiones. Incorporar el FR en las estimaciones de la IF no mejora la reproducibilidad ni la validez de las mismas.

Palabras clave: consumo de alimentos, estudios de validación, reproducibilidad de resultados, México
Galván-Portillo M,Torres-Sánchez L, Hernández-Ramírez RU,Anaya-Loyola, MA.

Validity and reproducibility of a food frequency questionnaire to estimate folate intake in a Mexican population.

Salud Publica Mex 20II; 53:237-246.

\section{Abstract}

Objective: To evaluate the reproducibility and validity of folate intake (Fl) estimated from a food frequency questionnaire (FFQ), taking into account the retention factor (RF) associated with meal preparation processes. Material and Methods: A total of 497 healthy adult participants were interviewed with a FFQ.A second interview was performed one year later of a sample of 100 randomly selected individuals who had not modified their diet during the previous year. In addition, serum folate (SF) concentrations were measured by radioimmunoassay to evaluate the validity of FI. Results: Reproducibility of IF was 0.36 and 0.34 for folate without FR and with FR, respectively. SF concentration significantly correlated with FI (0.18 with RF; 0.21 without RF, $p<0.05)$. Conclusions: Incorporating the folate RF in estimates of FI does not improve the reproducibility and validity of those estimates.

Key words: validation studies, reproducibility of results, folate, Mexico

(I) Centro de Investigación en Salud Poblacional, Instituto Nacional de Salud Pública, México

(2) Escuela de Nutrición. Facultad de Ciencias Naturales. Universidad Autónoma de Querétaro

Fecha de recibido: 20 de agosto de 2010 - Fecha de aceptado: 10 mayo de 2011 Solicitud de sobretiros: Dra. Marcia Galván Portillo. Instituto Nacional de Salud Pública. Av. Universidad No. 655 col. Santa María Ahuacatitlán, CP 62100, Cuernavaca, Morelos, México.

Correo electrónico: mgalvan@insp.mx 


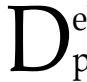
bido a que el folato aporta grupos metilo a los procesos epigenéticos involucrados en la correcta metilación del $\mathrm{ADN},{ }^{1}$ en la actualidad existe un creciente interés acerca del papel que juega su ingestión en la génesis de las enfermedades relacionadas con alteraciones en el metabolismo de un carbono, como son diversos tipos de cáncer, ${ }^{2-5}$ problemas cardiovasculares, ${ }_{,}^{6}$ defectos del tubo neural, ${ }^{7-9}$ enfermedades neurológicas, ${ }^{10}$ anemia, ${ }^{11,12}$ complicaciones durante el embarazo ${ }^{13-14} \mathrm{e}$ infertilidad masculina. ${ }^{15}$

Para estimar la ingestión crónica de folato, el cuestionario de frecuencia de consumo de alimentos (CFC) es uno de los instrumentos más empleados. Su validez no sólo depende de la representatividad de las porciones de alimentos fuentes de folato que se incluyan en el mismo, sino también de la forma de preparación y del tiempo de cocción de los alimentos. De acuerdo con los factores de retención (FR) estimados para el folato, la pérdida de este nutrimento varía entre $0 \%$ para cereales instantáneos, hasta 70\% en leguminosas hervidas, fritas $\mathrm{u}$ horneadas por un periodo de tiempo de 2 a 2.5 horas. ${ }^{16}$

Los estudios de validez del CFC para estimar el consumo de folato realizados hasta ahora no incorporan información relacionada con la forma de preparación de los alimentos y muestran gran variación en los resultados. Los coeficientes de correlación para la ingestión de folato (IF) obtenidos entre $\mathrm{CFC}$ versus registros dietéticos varían de 0.32 a $0.84,{ }^{17-24}$ de $0.26-0.98$ al compararlos con el recordatorio de 24 horas, ${ }^{25-32}$ de $0.22-0.67$ respecto del registro de pesos y medidas ${ }^{33-36}$ y de $0.11-0.94$ y $0.07-$ 0.95 en relación con los niveles de FS y eritrocitario, respectivamente. ${ }^{21,26,32,35-41}$ Por su parte, los estudios sobre reproducibilidad del folato a través del CFC han reportado coeficientes de correlación ajustados por energía, que varían desde 0.26 hasta $0.72,{ }^{17-19,22,24,25,29,39}$ lo cual se ha atribuido principalmente a las diferencias en el tiempo entre las aplicaciones de los cuestionarios.

El objetivo del presente estudio es evaluar la validez y la reproducibilidad de la IF a partir de un CFC de 127 alimentos, que considera el FR correspondiente al método de cocción más empleado en los alimentos fuentes de folato.

\section{Material y métodos}

Se llevó a cabo la validación de un CFC de alimentos en 497 individuos sanos, de ambos sexos, entre 21 y 88 años de edad, que participaron como controles durante 2004-2006 en un estudio de casos y controles sobre cáncer gástrico y dieta en la Ciudad de México y en el área conurbana, ${ }^{42}$ el cual contó con la aprobación del Comité de Ética del Instituto Nacional de Salud Pública.
Los criterios de elegibilidad de los controles fueron tener el mismo sexo y \pm 3 años la edad del caso índice, sin antecedentes de ningún tipo de cáncer y residencia permanente en la Ciudad de México y en el área conurbana. La identificación de los individuos se llevó a cabo a través del marco muestral maestro empleado en las encuestas nacionales de salud. La información detallada sobre dicha selección fue publicada previamente. ${ }^{43}$ Brevemente, los hogares fueron seleccionados probabilisticamente a partir de un listado de manzanas, y al interior de los mismos, se identificaron aleatoriamente los individuos elegibles.

\section{Cuestionario de frecuencia de consumo de alimentos}

El cuestionario empleado para la estimación de la IF diaria contiene 127 alimentos, ${ }^{44}$ agrupados en: lácteos, frutas, vegetales, carnes, carnes procesadas, pescados, refrescos, dulces, cereales y panes. Cada alimento tiene una porción predeterminada y 10 opciones de frecuencia de consumo, que van desde "Nunca" hasta "Seis veces al día". El consumo de frutas y verduras se ajustó de acuerdo con su disponibilidad en el mercado a lo largo del año, por ejemplo, la ciruela se encuentra disponible seis meses al año, por tanto, la frecuencia de consumo se dividió entre dos. Para fines de este reporte, el cuestionario se actualizó con una columna sobre la forma más frecuente de cocinar (hervido, al vapor, horneado, asado, en microondas, frito) los 82 alimentos considerados como fuentes de folato (hígado, lentejas, frijol, habas, chícharos, cereales, manzana, ciruela, plátano, naranja, espinaca, coliflor, brócoli, elote, etcétera).

\section{Estimación de la IF}

La IF en microgramos por día ( $\mu \mathrm{g} / \mathrm{d})$ se estimó con base en la frecuencia de consumo de los 82 alimentos fuentes de folato reportada durante el año previo por los participantes y el contenido de folato en los alimentos que se encuentra disponible en el paquete computarizado Food Intake Analysis System 3.0 (FIAS) (The University of Texas, Houston, 1996), a través de un programa de FoxPro.

La información nutricional de la base de datos del FIAS fue comparada y adecuada con los valores nutricionales de las tablas de composición de alimentos mexicanos desarrolladas por el Instituto Nacional de Nutrición en 1996, de acuerdo con la metodología descrita previamente. ${ }^{45}$ Además de la información acerca del contenido de folato y otros 27 nutrimentos, este paquete cuenta con la ventaja de que permite incluir el proceso de cocción utilizado y estimar a través del FR 
la proporción de folato que permanece en los alimentos cocidos en relación con la cantidad de nutrimento originalmente presente en los alimentos crudos.

\section{Folato en suero}

El folato sérico (FS) se determinó en la muestra de sangre $(5 \mathrm{ml})$ de cada participante. Las muestras se obtuvieron en vacutainers con EDTA, que fueron cubiertos con papel aluminio al momento de la toma, para evitar las pérdidas de folato por la acción de la luz. Todas las muestras se mantuvieron en refrigeración hasta el momento en que fueron centrifugadas para separar el suero de las células rojas. El suero se almacenó en viales de $1.5 \mathrm{ml}$, cubiertos con papel estaño y a $-70{ }^{\circ} \mathrm{C}$ hasta su análisis.

Los niveles FS se determinaron por duplicado con un ensayo in vitro que determina de manera simultánea folato y vitamina $\mathrm{B}_{12}$, mediante un radioinmunoensayo [(SimulTRAC Kit vitamina $B_{12}$ (Co57)/folato (I125)]. Es un ensayo competitivo en el que la vitamina $B_{12}$ y el folato sin marcar compiten con sus equivalentes marcados por el limitado número de lugares de unión disponibles, con lo que se reduce la cantidad ligada de vitamina $B_{12}$ o folato marcado.

El nivel de radioactividad es proporcional a la concentración en la muestra del paciente. El folato como ácido pteroilglutámico se utiliza como estándar y trazador en una mezcla de incubación a $\mathrm{pH}$ 9.3. En cada corrida se incluyeron muestras de suero como control de calidad (1, 2, 3 para concentraciones muy bajas, medianas y altas).

En cuanto a especificidad, el ácido 6-metiltetrahidrofólico y el pteroilglutámico tienen igual afinidad por el fijador en este ensayo y en cuanto a sensibilidad, definida como la concentración a $90 \%$ de fijación del trazador es $60 \mathrm{pg} / \mathrm{ml}$ para la $B_{12}$ y de $0.25 \mathrm{ng} / \mathrm{ml}$ para el folato. Estas mediciones se realizaron en los laboratorios de la Universidad de Davis (UCDavis, Lab).

\section{Validez y reproducibilidad}

La validez en la estimación de la IF se evaluó al comparar la información obtenida a través del CFC de los 497 individuos con sus respectivas concentraciones séricas de folato, considerado como estándar de oro.

La reproducibilidad de la IF se estimó en 100 individuos seleccionados aleatoriamente del total de sujetos, a quienes se les aplicó por duplicado el CFC, con una diferencia de un año entre cada aplicación y durante la misma estación del año. El criterio de elegibilidad para ser considerados en este subgrupo fue que no hubieran modificado su dieta en el año previo a la primera entrevista.

\section{Análisis estadístico}

Se realizó la prueba $t$ de Student o ji cuadrada para comparar las características de la población de estudio, según el género, incluyendo los niveles de FS en dos categorías: adecuado $(\geq 10 \mathrm{nmol} / \mathrm{l})$ versus deficiente $(<10 \mathrm{nmol} / 1){ }^{46}$

El consumo de folato, vitamina $\mathrm{B}_{12}, \mathrm{~B}_{6}$ y macronutrimentos se ajustó por residuales de energía, empleando el método de regresión residual sugerido por Willet y Stampfer ${ }^{47}$ y se compararon las medianas respectivas, con y sin FR según género, mediante la prueba no paramétrica de Wilcoxon para muestras pareadas.

La IF y los niveles de FS fueron normalizados mediante transformaciones logarítmicas. La reproducibilidad de la IF (entre la aplicación basal y la subsecuente) con y sin FR se evaluó a través de la prueba t pareada para las medias geométricas, el coeficiente de variación (CV), el coeficiente de correlación Intraclase (CCI) y método de Bland-Altman (BA). Por otro lado, el coeficiente de correlación de Pearson (CCP) y la regresión lineal simple y ajustada se estimaron para valorar tanto la reproducibilidad como la validez. Cada modelo fue ajustado por edad (años), escolaridad (años), tabaquismo (cajetillas/año), consumo de alcohol (gr/d), género e IMC. Los valores $p<0.05$ se consideraron como significativos. El análisis fue realizado con el software estadístico STATA 9.2.

\section{Resultados}

En el cuadro I se muestran las características seleccionadas de la población de estudio total y por género. La talla, el peso, los años de educación, el consumo de tabaco y alcohol fueron significativamente mayores en los hombres, comparados con las mujeres. En contraste, el índice de masa corporal y los niveles de FS fueron significativamente menores en los hombres en comparación con las mujeres. El 3.8\% de la población total presentó una deficiencia de folato $(<10 \mathrm{nmol} / 1)$. Estos resultados fueron similares en la muestra de los 100 individuos seleccionados para la evaluación de la reproducibilidad. Por ejemplo, la edad promedio de la muestra fue de $57 \pm 15$ años y no se observó diferencia significativa según genero; asimismo, el peso en promedio fue de $68.2 \pm 11.8$ $\mathrm{kg}$ y al estratificar por sexo, los hombres $(71.4 \pm 11.3 \mathrm{~kg}$ ) fueron significativamente más pesados que las mujeres $(64.8 \pm 11.5 \mathrm{~kg})$ (datos no presentados).

La estimación de la IF con el FR fue significativamente más baja, tanto en hombres como en mujeres, 


\section{Cuadro I \\ Características de la población. Estudio de validación y Reproducibilidad de la ingestión de folato, Según sexo. Ciudad de México, 2004-2006}

\begin{tabular}{|c|c|c|c|}
\hline Característica & $\begin{array}{c}\text { Todos } \\
(n=497)\end{array}$ & $\begin{array}{l}\text { Mujeres } \\
(n=234)\end{array}$ & $\begin{array}{c}\text { Hombres } \\
(n=263)\end{array}$ \\
\hline \multicolumn{4}{|l|}{ Edad (años) } \\
\hline Medias $\pm \mathrm{DE}$ & $59 \pm 14$ & $58 \pm 15$ & $59 \pm 14$ \\
\hline \multicolumn{4}{|l|}{ Talla (m) } \\
\hline Medias $\pm \mathrm{DE}$ & $1.59 \pm 0.1$ & $1.53 \pm 0.1$ & $1.65 \pm 0.1^{*}$ \\
\hline \multicolumn{4}{|l|}{ Peso (kg) } \\
\hline Medias $\pm \mathrm{DE}$ & $69.2 \pm 12.7$ & $65.4 \pm 11.3$ & $72.5 \pm 12.9 *$ \\
\hline \multicolumn{4}{|l|}{ Índice de masa corporal } \\
\hline Medias $\pm \mathrm{DE}$ & $27.2 \pm 4.3$ & $27.9 \pm 4.5$ & $26.6 \pm 4 . I^{*}$ \\
\hline \multicolumn{4}{|l|}{ Educación (años) } \\
\hline Medias $\pm \mathrm{DE}$ & $6 \pm 4$ & $5 \pm 4$ & $6 \pm 5^{*}$ \\
\hline \multicolumn{4}{|l|}{ Tabaquismo (cajetillas por año) } \\
\hline No fumadores (\%) & 48.9 & 71.4 & 28.6 \\
\hline Medias $\pm \mathrm{DE}$ & $139 \pm 161$ & $107 \pm 116$ & $147 \pm|7| *$ \\
\hline \multicolumn{4}{|l|}{ Consumo de alcohol (gr/d) } \\
\hline No consumidores (\%) & 65.2 & 77.3 & 54.4 \\
\hline Medias $\pm \mathrm{DE}$ & $2.8 \pm 4.5$ & $1.3 \pm 2.5$ & $3.5 \pm 5.1 *$ \\
\hline \multicolumn{4}{|l|}{ Folato sérico (nmol/l) } \\
\hline Medias $\pm \mathrm{DE}$ & $22.7 \pm 7.9$ & $24.1 \pm 7.6$ & $21.5 \pm 8.1^{*}$ \\
\hline$<10 \mathrm{nmol} / \mathrm{l}(\%)$ & 3.8 & 2.6 & 4.9 \\
\hline
\end{tabular}

* $p<0.05$, DE: desviación estándar prueba t de Student (variables cuantitativas) ○ $\chi^{2}$ (variables categóricas), para la comparación entre sexos

en comparación con los valores obtenidos sin el FR. En contraste, la estimación del consumo de macronutrimentos, vitaminas $\mathrm{B}_{12} \mathrm{y}_{6}$ no resultó afectada por la consideración del FR (cuadro II).

Las medias geométricas de la IF (cuadro III), con y $\sin \mathrm{FR}$, fueron estadísticamente mayores en la entrevista basal comparadas con la segunda entrevista, tanto en el grupo de hombres $(291.93 \pm 1.28$ vs. $227.78 \pm 1.28$ sin FR; $283.99 \pm 1.27$ vs. $220.97 \pm 1.29$ con FR), como en el de mujeres (313.82 \pm 1.24 vs. $245 \pm 1.26$ sin FR; $303.78 \pm 1.22$ vs. $239.88 \pm 1.28$ con FR), con un CV no significativo de aproximadamente $30 \%$, respectivamente.

En la reproducibilidad, se observó una correlación moderada entre las estimaciones del folatos con unos CCI que variaron de 0.34-0.39 para la IF sin FR en toda la población o de acuerdo con el género. Para IF con FR los CCI fluctuaron entre 0.33-0.35. Con respecto a los CCP, se observaron correlaciones significativas sólo entre las estimaciones de IF considerando el FR $(0.36, p=0.0190)$ de toda la población. Al estratificar por género, solo en el grupo masculino se observaron CCP estadísticamente significativos, independientemente del FR (cuadro III).
Las medias de la diferencia (MD) de las estimaciones de folato de la entrevista basal con las de las segundas entrevistas y los límites de concordancia (LCC) obtenidos del análisis de BA, se presentan en el cuadro III. Se observó que el error sistemático en la medición de folato del primer $\mathrm{CFC}$ respecto del segundo, varía entre 27 y $28 \%$. Los gráficos de BA, según FR y género se presentan en la figura IA-IF. En las estimaciones de folato, sin FR y con FR, podemos observar que el cuestionario basal tiende a subestimar ligeramente la IF y esta subestimación parece ser mayor entre los que reportan una mayor IF (figuras 1A, 1B). Esta tendencia en el subreporte de la IF se mantiene al estratificar por sexo, siendo más evidente para el folato con FR en el grupo de mujeres (figura 1 E); por el contrario, en los hombres se puede observar que la subestimación de folato es muy pequeña (figura 1D y $1 G$ ).

En relación con la validez del instrumento (cuadro IV), se observó una asociación significativa de la IF ajustada por energía, edad, consumo de alcohol, tabaquismo, educación e IMC con los niveles de FS, independientemente del FR, sin embargo, la significancia no se mantuvo al estratificar por género. 


\section{CUADRO II \\ CONSUMO DIARIO DE MACRONUTRIMENTOS, FOLATO, VITAMINA B 12 Y B 6 DE LA POBLACIÓN EN ESTUDIO DE VALIDACIÓN Y REPRODUCIBILIDAD DE LA INGESTIÓN DE FOLATO, DE ACUERDO CON FACTOR DE RETENCIÓN Y SEXO. \\ Ciudad de México, 2004-2006}

\begin{tabular}{|c|c|c|c|c|c|c|}
\hline \multirow{2}{*}{ Nutrimento/día } & \multicolumn{2}{|c|}{$\begin{array}{c}\text { Todos } \\
(n=497)\end{array}$} & \multicolumn{2}{|c|}{$\begin{array}{l}\text { Mujeres } \\
(n=234)\end{array}$} & \multicolumn{2}{|c|}{$\begin{array}{l}\text { Hombres } \\
(n=263)\end{array}$} \\
\hline & & & \multicolumn{4}{|c|}{ Factor de retención } \\
\hline & $\operatorname{Sin}$ & Con & $\operatorname{Sin}$ & Con & $\operatorname{Sin}$ & Con \\
\hline \multicolumn{7}{|l|}{ Energía total(kcal) } \\
\hline Mediana & 2127.2 & $2|5| .8$ & 2155.6 & 2147.3 & 2114.7 & 2153.3 \\
\hline Percentiles 25-75 & I 697.2-2 565.2 & | 758.2-2 595.8 & $1719.4-2530.9$ & | 782.8-2 576.6 & | 645.7-2 591.0 & I 736.8-2 634.9 \\
\hline \multicolumn{7}{|l|}{ Proteína (g) } \\
\hline Mediana & 68.0 & 69.6 & 69.5 & 70.9 & 67.3 & 68.9 \\
\hline Percentiles 25-75 & $64.1-72.8$ & $65.9-74.4$ & $65.4-74.0$ & $67.2-75.4$ & $62.8-71.4$ & $65.1-73.2$ \\
\hline \multicolumn{7}{|c|}{ Hidratos de carbono(g) } \\
\hline Mediana & 313.5 & 313.7 & 313.1 & 313.3 & 313.7 & 314.8 \\
\hline Percentiles 25-75 & $299.9-326.3$ & $296.9-326.6$ & $299.0-325.9$ & $294.8-326.4$ & $299.9-326.6$ & $297.2-327.5$ \\
\hline \multicolumn{7}{|l|}{ Grasa total $(\mathrm{g})$} \\
\hline Mediana & 80.4 & 84.6 & 80.6 & 85.2 & 80.1 & 84.3 \\
\hline Percentiles 25-75 & $75.8-85.4$ & 79.9-91.1 & $76.6-86.6$ & $80.4-92.4$ & $75.3-84.3$ & $79.5-90.3$ \\
\hline \multicolumn{7}{|l|}{ Folato $(\mathrm{mg})^{*}$} \\
\hline Mediana & 277.1 & 265.0 & 289.1 & 275.9 & 267.7 & 256.7 \\
\hline Percentiles 25-75 & $242.5-320.8$ & $231.8-308.6$ & $252.6-327.2$ & $242.7-315.1$ & $234.7-313.2$ & $222.8-302.8$ \\
\hline \multicolumn{7}{|l|}{ Vitamina $B_{12}(\mathrm{mg})$} \\
\hline Mediana & 3.8 & 4.0 & 3.8 & 4.1 & 3.74 & 3.9 \\
\hline Percentiles 25-75 & $2.6-5.1$ & $2.8-5.4$ & $2.7-5.5$ & $2.9-5.8$ & $2.5-4.9$ & $2.7-5.3$ \\
\hline \multicolumn{7}{|l|}{ Vitamina $B_{6}(\mathrm{mg})$} \\
\hline Mediana & 1.5 & 1.5 & 1.5 & 1.5 & 1.5 & 1.5 \\
\hline Percentiles $25-75$ & $1.3-1.7$ & $1.3-1.7$ & $1.4-1.7$ & $1.3-1.7$ & $1.3-1.7$ & $1.3-1.7$ \\
\hline
\end{tabular}

\section{Discusión}

El presente estudio reporta la reproducibilidad y la validación de un CFC para estimar la IF que considera las pérdidas de folato que experimenta el alimento durante los procesos de preparación para su consumo a través del FR. Los resultados sugieren que la incorporación del FR no incrementa la reproducibilidad de la estimación de folato obtenida a través del CFC, y tampoco mejora la validez del mismo. Sin embargo, fue evidente que para estimar la IF de manera más puntual, es necesario estratificar por sexo.

Para la validación, se empleó como método de comparación un biomarcador de la IF, ya que una de sus ventajas respecto de los métodos de medición de consumo de alimentos (recordatorio de 24 horas o registro dietético), es que da una medición objetiva del consumo de nutrimento de interés, y sus errores de medición son independientes de los del CFC ${ }^{48}$

En el caso particular del FS, éste ha sido empleado en un número reducido de estudios como estándar de oro contra el CFC, ${ }^{49}$ su uso es debatible, ya que dichos niveles reflejan una ingestión reciente, mientras que el CFC se asocia con un consumo de folato crónico, esta situación explica parcialmente los bajos CCP obtenidos por éste $(0.21)$ y otros estudios $(0.42,0.36,0.20$ 0.16). $32,39,40,50$

Por su parte, la reproducibilidad de la IF, considerando el FR (0.32), observada entre las mujeres, es similar a lo reportado por Munger et al. ${ }^{25}(0.33)$, quien aplicó por duplicado en un periodo de dos años un CFC a mujeres de 55 a 69 años. Respecto de los hombres, sólo 


\section{CuAdro III \\ REPRODUCIBILIDAD DEL CONSUMO DIARIO DE FOLATO DE ACUERDO CON FACTOR DE RETENCIÓN

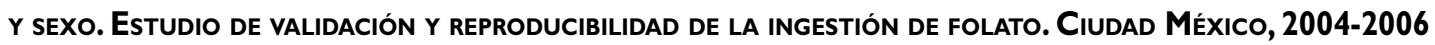

\begin{tabular}{|c|c|c|c|c|}
\hline \multirow{3}{*}{ Consumo de folato* (mcg/día) } & \multicolumn{4}{|c|}{ Factor de retención } \\
\hline & \multicolumn{2}{|c|}{$\operatorname{Sin}$} & \multicolumn{2}{|c|}{ Con } \\
\hline & ${ }^{\ddagger}$ Media + DE & Min - Max & $¥$ Media + DE & Min - Max \\
\hline \multicolumn{5}{|l|}{ Todos $(n=100)$} \\
\hline Cuestionario basal & $302.26 \pm 1.23$ & 158.67-554.07 & $293.33 \pm 1.25$ & $|53.17-50| .32$ \\
\hline Segundo cuestionario & $235.96 \pm 1.27$ & || $7.5|-4| 4.9 \mid$ & $229.85 \pm 1.29$ & $1|7.48-4| 5.93$ \\
\hline$t$ de Student, valor $p$ & \multicolumn{2}{|c|}{$<0.000$} & \multicolumn{2}{|c|}{$<0.000$} \\
\hline Coeficiente de variación (\%) & \multicolumn{2}{|c|}{30.61} & \multicolumn{2}{|c|}{30.12} \\
\hline $\begin{array}{l}\text { Coeficiente correlación intraclase } \\
(95 \% \mathrm{IC})\end{array}$ & \multicolumn{2}{|c|}{$0.36(0.0-1.0)$} & \multicolumn{2}{|c|}{$0.34(0.0-0.9)$} \\
\hline $\begin{array}{l}\text { Coeficiente correlación Pearson } \\
\text { crudo*, valor } p \\
\text { ajustado, } \$ \text { valor } p\end{array}$ & \multicolumn{2}{|c|}{$0.32,0.0599$} & \multicolumn{2}{|c|}{$0.36,0.0190$} \\
\hline $\begin{array}{l}\text { Regresión lineal }(\beta) \\
\text { cruda*, valor } p \\
\text { ajustada, }{ }^{\S} \text { valor } p\end{array}$ & \multicolumn{2}{|c|}{$\begin{array}{l}0.21,0.022 \\
0.19,0.034\end{array}$} & \multicolumn{2}{|c|}{$0.25,0.004$} \\
\hline $\begin{array}{l}\text { Análisis de Bland-Altman } \\
\text { MD (anti log) } \\
95 \% \text { LCC (anti log) }\end{array}$ & \multicolumn{2}{|c|}{$\begin{array}{c}0.248(1.28) \\
-0.3 \mid 4 ; 0.810(0.73 ; 2.24)\end{array}$} & \multicolumn{2}{|c|}{$\begin{array}{c}0.244(I .27) \\
-0.310 ; 0.798(0.73 ; 2.22)\end{array}$} \\
\hline \multicolumn{5}{|l|}{ Mujeres $(n=48)$} \\
\hline Cuestionario basal & $313.86 \pm 1.24$ & |78.83-483.49 & $303.78 \pm 1.22$ & I78.52-479.33 \\
\hline Segundo cuestionario & $245.16 \pm 1.26$ & $|43.40-4| 4.9 \mid$ & $239.88 \pm 1.28$ & $|32.9|-4 \mid 5.93$ \\
\hline$t$ de student, valor $p$ & \multicolumn{2}{|c|}{$<0.000$} & \multicolumn{2}{|c|}{$<0.000$} \\
\hline $\begin{array}{l}\text { Coeficiente de variación (\%) } \\
\text { Coeficiente correlación intraclase } \\
(95 \% \mathrm{IC})\end{array}$ & \multicolumn{2}{|c|}{$\begin{array}{c}30.45 \\
0.39(0.0-1 . I)\end{array}$} & \multicolumn{2}{|c|}{$\begin{array}{c}31.14 \\
0.35(0.0-1.0)\end{array}$} \\
\hline $\begin{array}{l}\text { Coeficiente correlación Pearson } \\
\text { crudo*, valor } p \\
\text { ajustado," valor } p\end{array}$ & \multicolumn{2}{|c|}{$\begin{array}{c}0.15,0.323 \\
0.25,0.5338\end{array}$} & & \\
\hline $\begin{array}{l}\text { Regresión lineal }(\beta) \\
\text { cruda*, valor } p \\
\text { ajustada, }{ }^{\#} \text { valor } p\end{array}$ & \multicolumn{2}{|c|}{$0.16,0.249$} & \multicolumn{2}{|c|}{$0.19,0.107$} \\
\hline $\begin{array}{l}\text { Análisis de Bland-Altman } \\
\text { MD (anti log) } \\
95 \% \text { LCC (anti log) }\end{array}$ & \multicolumn{2}{|c|}{$\begin{array}{c}0.247(1.28) \\
-0.314-0.808(0.73-2.24)\end{array}$} & -0.317 & $3-2.20)$ \\
\hline Hombres $(n=52)$ & & & & \\
\hline Cuestionario basal & $291.93 \pm 1.28$ & I58.67-554.07 & $283.99 \pm 1.27$ & $|53.17-50| .32$ \\
\hline Segundo cuestionario & $227.78 \pm 1.28$ & || $7.5 \mid-367.97$ & $220.97 \pm 1.29$ & II 7.48-352.44 \\
\hline$t$ de student, valor $p$ & & & & \\
\hline $\begin{array}{l}\text { Coeficiente de variación (\%) } \\
\text { Coeficiente correlación intraclase } \\
(95 \% \mathrm{IC})\end{array}$ & & & & \\
\hline $\begin{array}{l}\text { Coeficiente correlación Pearson } \\
\text { crudo*, valor } p \\
\text { ajustado," valor p }\end{array}$ & & & & \\
\hline $\begin{array}{l}\text { Regresión lineal }(\beta) \\
\text { cruda*, valor } p \\
\text { ajustada," valor } p\end{array}$ & & & & \\
\hline $\begin{array}{l}\text { Análisis de Bland-Altman } \\
\text { MD (anti log) } \\
95 \% \text { LCC (anti log) }\end{array}$ & -0.32 & $3-2.26)$ & -0.308 & $3-3.59)$ \\
\hline
\end{tabular}

* Ajustado por residuales de energía

¥ Medias geométricas

§Ajustado por edad (años), consumo de alcohol (gr/d), tabaquismo (número de cajetillas por año), educación (años), índice de masa corporal y género \# Ajustado por edad (años), consumo de alcohol (gr/d), tabaquismo (número de cajetillas por año), educación (años), índice de masa corporal DE: desviación estándar; MD: medias de la diferencia de folato de la entrevista basal vs $2^{\mathrm{a}}$. entrevista; LC: límites de concordancia 


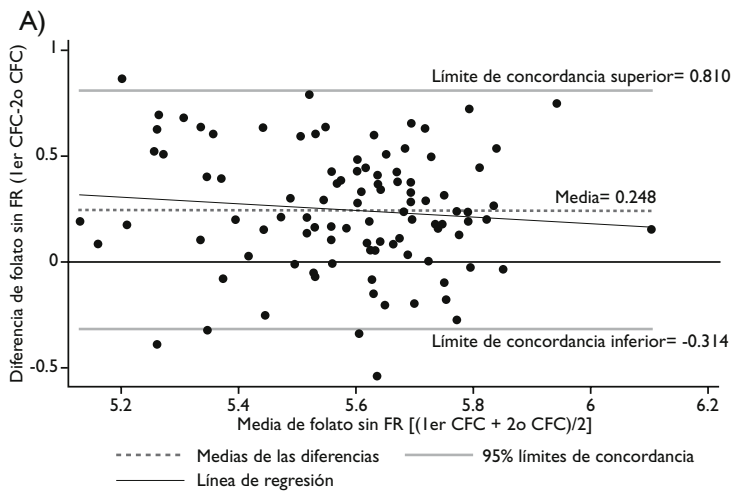

$y=0$ es la línea de concordancia promedio perfecta
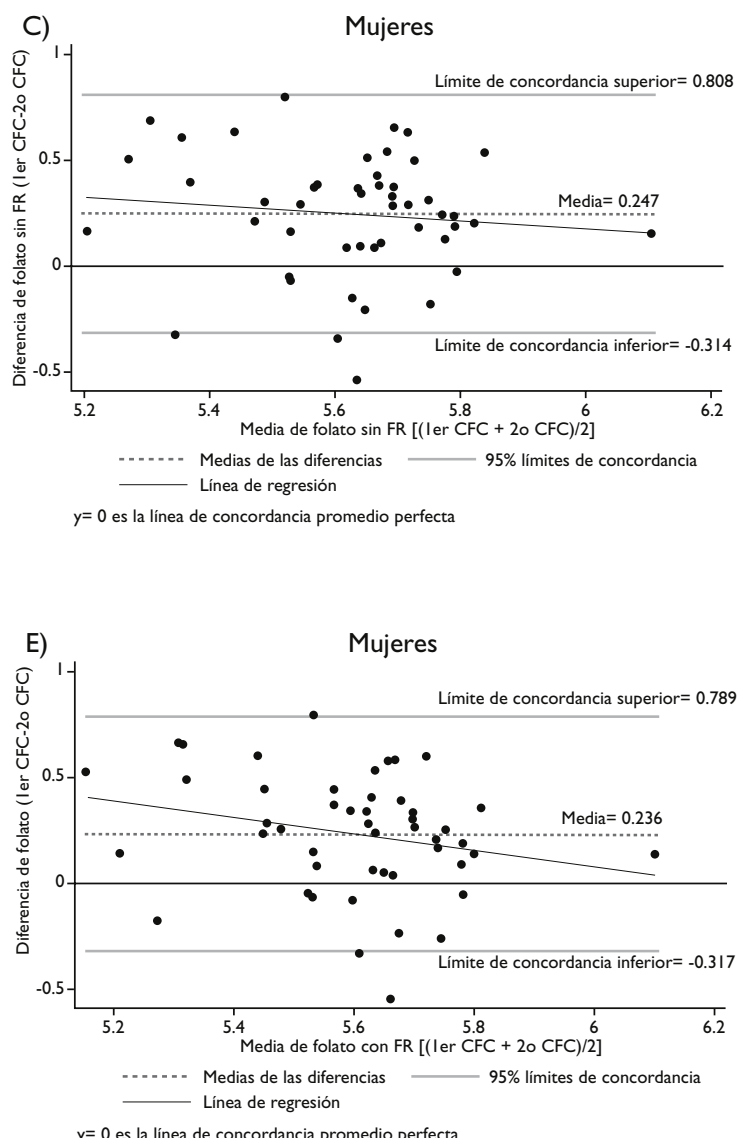

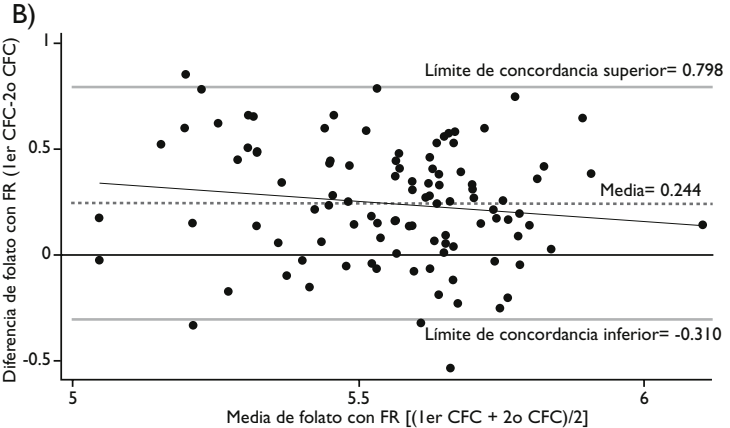

Media de folato con FR [(ler CFC $+20 \mathrm{CFC}) / 2]$

Medias de las diferencias $\quad 95 \%$ límites de concordancia

L Línea de regresión

$y=0$ es la línea de concordancia promedio perfecta
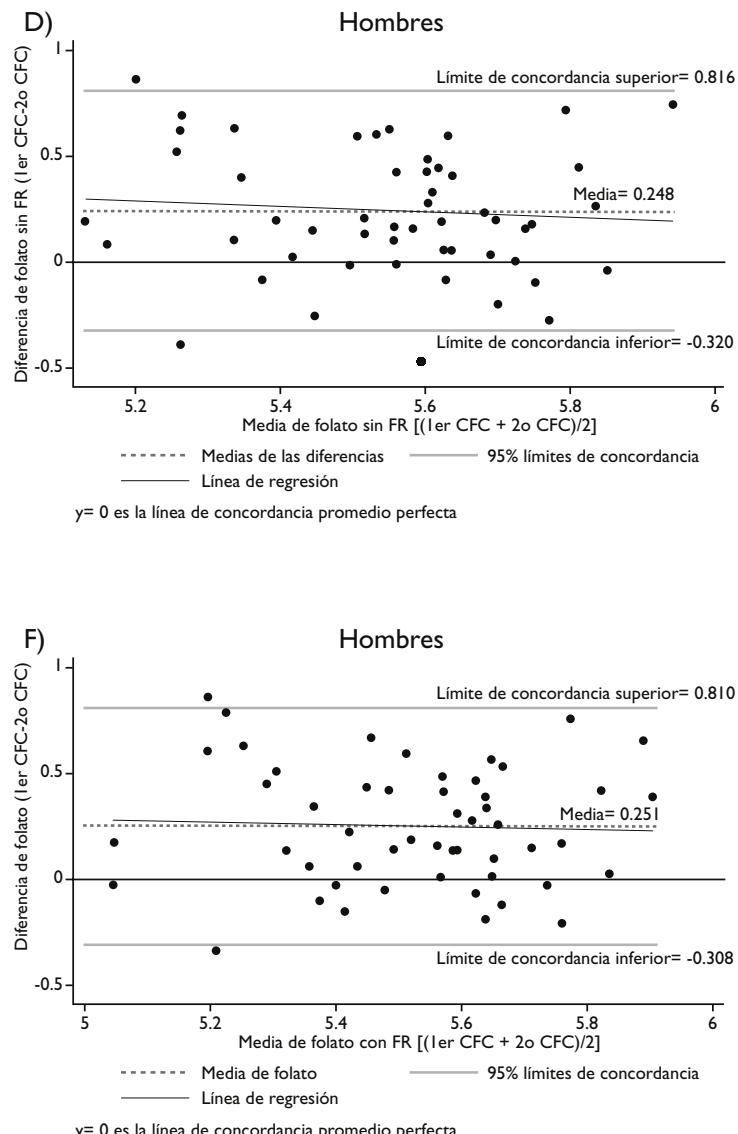

$y=0$ es la línea de concordancia promedio perfecta
A) Toda la población de estudio con factor de retención (FR)

B) Toda la población de estudio sin FR
Sin FR C) Mujeres, D) Hombres

Con FR E) Mujeres, F) Hombres

Figura i. Gráficos de Bland-Altman para la diferencia logarítmica de las estimaciones de consumo de folato entre el PRIMERO Y EL SEGUNDO CUESTIONARIO DE FRECUENCIA DE CONSUMO (CFC), DE ACUERDO CON FACTOR DE RETENCIÓN (FR) Y SEXo. CiUdAD de MéXICO, 2004-2006 


\section{Cuadro IV

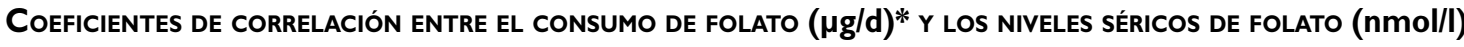 Según factor de retención (FR) y SeXo. Ciudad de MéXico, 2004-2206}

\begin{tabular}{|c|c|c|c|c|c|c|c|}
\hline Folato & & $\begin{array}{c}\text { Coeficiente } \\
\text { de correlación } \\
\text { crudo }\end{array}$ & Valor $p$ & $\begin{array}{c}\text { Coeficiente } \\
\text { de correlación } \\
\text { ajustado }\end{array}$ & Valor $p$ & $\beta$ & Valor $p$ \\
\hline \multirow{3}{*}{ Dietético vs. sérico } & Sin FR & 0.11 & 0.02 & $0.21^{\ddagger}$ & 0.0075 & 0.07 & 0.018 \\
\hline & & & & & & & \\
\hline & Con FR & 0.09 & 0.04 & $0.18^{\ddagger}$ & 0.0467 & 0.06 & 0.043 \\
\hline \multicolumn{8}{|l|}{ Mujeres $(n=234)$} \\
\hline \multirow{3}{*}{ Dietético vs. sérico } & Sin FR & 0.10 & 0.13 & $0.18^{\S}$ & 0.3759 & 0.06 & 0.13 \\
\hline & & & & & & & \\
\hline & Con FR & 0.06 & 0.33 & $0.18^{\S}$ & 0.3359 & 0.04 & 0.335 \\
\hline \multicolumn{8}{|l|}{ Hombres $(n=263)$} \\
\hline \multirow{2}{*}{ Dietético vs. sérico } & Sin FR & 0.07 & 0.25 & $0.20^{\S}$ & 0.1296 & 0.04 & 0.253 \\
\hline & Con FR & 0.07 & 0.26 & $0.17^{\S}$ & 0.3294 & 0.04 & 0.257 \\
\hline
\end{tabular}

\footnotetext{
* Ajustado por residuales de energía

¥Ajustado por edad (años), consumo de alcohol (gr/d), tabaquismo (número de cajetillas por año), educación (años), IMC y género

$\S$ Ajustado por edad (años), consumo de alcohol (gr/d), tabaquismo (número de cajetillas por año), educación (años), IMC
}

dos estudios reportan coeficientes de correlación en este grupo $\left(0.77,{ }^{39} 0.63^{17}\right)$ y ambos son mayores al observado en este estudio (0.48).

En otros estudios se han obtenido coeficientes mayores a los nuestros $\left(0.58,{ }^{18} 0.69^{22}\right.$ y $\left.0.72^{19}\right)$, y probablemente esto se deba a diferencias en la composición por género y edad de las poblaciones de estudio, que se puede asociar con cambios en los hábitos dietéticos; por ejemplo, los adultos mayores tienden a modificar menos la dieta y producir mejores corelaciones. ${ }^{51}$ Otra posible explicación es que haya diferencias en la estructura de los cuestionarios en función del número de alimentos y porciones, y en particular, al tiempo transcurrido entre las dos entrevistas que se relaciona inversamente con la magnitud de los coeficientes. ${ }^{51}$

Entre las limitaciones metodológicas a considerar en la interpretación de estos resultados es importante mencionar que no se obtuvo información acerca del uso de suplementos y/o complementos alimenticios con folato. De acuerdo con la Encuesta Nacional de Nutrición 1999, se estima que $18 \%$ de mujeres en edad reproductiva consume suplementos por un periodo no mayor a dos meses, ${ }^{52}$ y algunos estudios sugieren que dicho consumo es mucho menor en el sexo masculino. ${ }^{53}$ Ante esta situación es posible que la inclusión del con- sumo de suplementos del folato en el CFC no hubiera incrementado significativamente la estimación de la IF ni los CCP respectivos.

Por otra parte, entre las fortalezas metodológicas, la posibilidad de un error diferencial que hubiera sesgado los estimadores reportados en cualquier dirección es prácticamente nula, debido a que se trata de un estudio ciego, tanto para los entrevistados, los entrevistadores y el personal que realizó las determinaciones de FS. Adicionalmente, se controló en el diseño por los cambios en la dieta que suceden con las variaciones estacionales, al realizar la entrevista basal a lo largo de un periodo de un año. Con la finalidad de descartar la posibilidad de un sesgo de selección, se comparó la prevalencia de no fumadores en la población total de estudio (48.9\%) y por género (hombres $28.6 \%$; mujeres $71.4 \%$ ) con los datos reportados por la Encuesta Nacional de Adicciones 2008 (ENA 2008), 54 para adultos de 18 y 65 años de edad en la Ciudad de México y no se encontró diferencia significativa entre las prevalencias de no fumadores de la población total $(46.5 \%)$, como tampoco entre el grupo de hombres (31.8\%). Sin embargo, entre las mujeres se observó una frecuencia de no fumadoras significativamente mayor en nuestra muestra en comparación con lo reportado por la ENA 2008 (59.3\%). 


\section{Agradecimientos}

Los autores agradecen a la licenciada Verónica López por la coordinación logística del trabajo de campo. Este trabajo fue financiado por el Consejo Nacional de Ciencia y Tecnología de México/Conacyt (Salud-2002 $-001-7107)$.

Conflictos de intereses: Los autores declararon no tener conflicto de intereses.

\section{Referencias}

I. Davis C, Uthus E. DNA methylation, cancer susceptibility and nutrient interactions. Exp Biol Med 2004;229:988-995.

2. Collin SM, Metcalfe C, Refsum H, Lewis SJ, Zuccolo L, Smith GD, et al. Circulating folate, vitamin $B_{12}$, homocysteine, vitamin $B_{12}$ transport proteins, and risk of prostate cancer: a case-control study, systematic review, and metaanalysis. Cancer Epidemiol Biomarkers Prev 20 10;19:1632-1642.

3. Xu X, Chen J. One-carbon metabolism and breast cancer: an epidemiological perspective.J Genet Genomics 2009;36:203-214.

4. De Klerk N, Milne E. Overview of recent studies on childhood leukemia, intra-uterine growth and diet. Radiat Prot Dosimetry 2008;132:255-258.

5. Hubner RA. Houlston RS. Folate and colorectal cancer prevention. $\mathrm{Br} J$ Cancer 2009; 100:233-239.

6. Wierzbicki AS. Homocysteine and cardiovascular disease: a review of the evidence. Diab Vasc Dis Res 2007;4: I43-I50.

7. Blencowe $\mathrm{H}$, Cousens S, Modell B, Lawn J. Folic acid to reduce neonatal mortality from neural tube disorders. Int J Epidemiol 2010;39:1 I0-121. 8. Wolff T, Witkop CT, Miller T, Syed SB. US Preventive Services Task Force. Folic acid supplementation for the prevention of neural tube defects: an update of the evidence for the US Preventive Services Task Force. Ann Intern Med 2009;150:632-639.

9. Blom HJ. Folic acid, methylation and neural tube closure in humans. Birth Defects Res A Clin Mol Teratol 2009;85:295-302.

I0. Stanger O, Fowler B, Piertzik K, Huemer M, Haschke-Becher E, Semmler $A$, et al. Homocysteine, folate and vitamin $B_{12}$ in neuropsychiatric diseases: review and treatment recommendations. Expert Rev Neurother 2009;9:1393-1412.

II. Cordero JF, Do A, Berry RJ. Review of interventions for the prevention and control of folate and vitamin $B_{12}$ deficiencies. Food Nutr Bull 2008;29:SI88-SI95. 12. Metz J.A high prevalence of biochemical evidence of vitamin $B_{12}$ or folate deficiency does not translate into a comparable prevalence of anemia. Food Nutr Bull 2008;29:S74-S85.

13. Molloy AM, Kirke PN, Brody LC, Scott JM, Mills JL. Effects of folate and vitamin $B_{12}$ deficiencies during pregnancy on fetal, infant, and child development. Food Nutr Bull 2008;29:SIOI-SIII.

14. Shah PS, Ohlsson A. Knowledge Synthesis Group on Determinants of Low Birth Weight and Preterm Births. Effects of prenatal multimicronutrient supplementation on pregnancy outcomes: a meta-analysis. CMAJ 2009;180:E99-EI08.

15. Forges T, Monnier-Barbarino P,Alberto JM, Guéant-Rodriguez RM, Daval JL, Guéant JL. Impact of folate and homocysteine metabolism on human reproductive health. Hum Reprod Update 2007;13:225-238.

16. Nutrient Data Laboratory, Beltsville Human Nutrition Research Center,Agricultural Research Service, US Department of Agriculture. USDA Table of nutrient retention factors [monografía en internet]. Release 5 2003. Disponible en: http://www.ars.usda.gov/nutrientdata (consultado: 8 marzo 20II).
17. Rimm EB, Giovannucci EL, Stampfer MJ, Colditz GA, Litin LB, Willett WC. Reproducibility and validity of an expanded self-administered semi quantitative food frequency questionnaire among male health professionals. Am J Epidemiol 1992; |35: I | |4- I 26.

I8. Longnecker MP, Lissner L, Holden JM, Flack VF, Taylor PR, Stampfer MJ, et al. The reproducibility and validity of a self-administered semi-quantitative food frequency questionnaire in subjects from South Dakota and Wyoming. Epidemiology 1993;4:356-365.

19. Friis S, Kjaer S, Stripp C, Overvad K. Reproducibility and relative validity of a self-administered semi quantitative food frequency questionnaire applied to younger women.J Clin Epidemiol 1997;50:303-3II

20. French MR, Langdon C, Levy-Milne R. Development of a validated food frequency questionnaire to determine folate intake. Can J Diet Pract Res 200I;62:82-86

2I. Yen J, Zoumas-Morse C, Pakiz B, Rock CL. Folate intake assessment: validation of a new approach. J Am Diet Assoc 2003; 103:991-1000.

22. Goulet J, Nadeau G, Lapointe A, Lamarche B, Lemieux S. Validity and reproducibility of an interviewer-administered food frequency questionnaire for healthy French-Canadian men and women. Nutr J 2004;3:13.

23. Shatenstein B, Nadon S, Godin C, Ferland G. Development and validation of a food frequency questionnaire. Can J Diet Pract Res 2005;66:6775.

24. Erkkola M, Karppinen M, Javanainen J, Räsänen L, Knip M,Virtanen SM. Validity and reproducibility of a food frequency questionnaire for pregnant Finnish women. Am J Epidemiol 200I;154:466-476.

25. Munger RG, Folsom AR, Kushi LH, Kaye SA, Sellers TA. Dietary assessment of older lowa women with a food frequency questionnaire: nutrient intake, reproducibility, and comparison with 24-hour dietary recall interviews. Am J Epidemiol 1992;136:192-200.

26. Wei EK, Gardner J, Field AE, Rosner BA, Colditz GA, Suitor CW. Validity of a food frequency questionnaire in assessing nutrient intakes of lowincome pregnant women. Matern Child Health J 1999;3:24I-246.

27. Knutsen SF, Fraser GE, Linsted KD, Beeson WL, Shavlik DJ. Comparing biological measurements of vitamin C, folate, alpha-tocopherol and carotene with 24-hour dietary recall information in nonhispanic blacks and whites. Ann Epidemiol 200 I; I l:406-4I6.

28. Kumanyika SK, Mauger D, Mitchell DC, Phillips B, Smiciklas-Wright H, Palmer JR. Relative validity of food frequency questionnaire nutrient estimates in the Black Women's Health Study.Ann Epidemiol 2003; I3: I I-I I8. 29. Bacardí-Gascón M, Ley y de Góngora S, Castro-Vázquez BY, JiménezCruz A. Validation of a semi quantitative food frequency questionnaire to assess folate status. Results discriminate a high-risk group of women residing on the Mexico-US border. Arch Med Res 2003;34:325-330.

30. Sevak L, Mangtani P, McCormack V, Bhakta D, Kassam-Khamis T, Dos Santos Silva I. Validation of a food frequency questionnaire to assess macroand micro-nutrient intake among South Asians in the United Kingdom. Eur J Nutr 2004;43:160-168.

3I. Segovia-Siapco G, Singh P, Jaceldo-Siegl K, Sabaté J. Validation of a food-frequency questionnaire for measurement of nutrient intake in a dietary intervention study. Public Health Nutr 2007; 10: I77-I84.

32. Verkleij-Hagoort AC, de Vries JH, Stegers MP, Lindemans J, Ursem NT, Steegers-Theunissen RP. Validation of the assessment of folate and vitamin $B_{12}$ intake in women of reproductive age: the method of triads. Eur J Clin Nutr 2007:61:610-615.

33. Flood VM, Smith WT,Webb KL, Mitchell P. Issues in assessing the validity of nutrient data obtained from a food-frequency questionnaire: folate and vitamin $B_{12}$ examples. Public Health Nutr 2004;7:751-756.

34. Ishihara J,Yamamoto S, Iso H, Inoue M, Tsugane S; JPHC FFQ Validation Study Group. Validity of a self-administered food frequency questionnaire (FFQ) and its generalizability to the estimation of dietary folate intake in Japan. Nutr J 2005 5;4:26.

35. Mikkelsen TB, Osler M, Olsen SF. Validity of protein, retinol, folic acid and $n-3$ fatty acid intakes estimated from the food-frequency questionnaire used in the Danish National Birth Cohort. Public Health Nutr 2006;9:77I-778. 
36. Brantsaeter AL, Haugen M,Alexander J, Meltzer HM. Validity of a new food frequency questionnaire for pregnant women in the Norwegian Mother and Child Cohort Study (MoBa). Matern Child Nutr 2008;4:28-43.

37. Jacques PF, Sulsky SI, Sadowski JA, Phillips JC, Rush D, Willett WC. Comparison of micronutrient intake measured by a dietary questionnaire and biochemical indicators of micronutrient status. Is J Clin Nutr 1993;57:182-189. 38. Green TJ,Allen OB, O'Connor DL. A three-day weighed food record and a semi quantitative food-frequency questionnaire are valid measures for assessing the folate and vitamin B-12 intakes of women aged 16 to 19 years.J Nutr 1998;128:1665-167।.

39. Pufulete M, Emery PW, Nelson M, Sanders TA. Validation of a short food frequency questionnaire to assess folate intake. Br J Nutr 2002;87:383-390. 40. Van de Rest O, Durga J,Verhoef P, Melse-Boonstra A, Brants HA. Validation of a food frequency questionnaire to assess folate intake of Dutch elderly people. Br J Nutr 2007;98:1014-1020.

4I. Owens JE, Holstege DM, Clifford AJ. Comparison of two dietary folate intake instruments and their validation by RBC folate. J Agric Food Chem 2007;55:3737-3740.

42. Galván-Portillo MV, Cantoral A, Oñate-Ocaña LF, Chen J, HerreraGoepfert R, Torres-Sanchez L, et al. Gastric cancer in relation to the intake of nutrients involved in one-carbon metabolism among MTHFR 677 TT carriers. Eur J Nutr 2009;48:269-276.

43. Tapia-Conyer R, Gutiérrez G, Sepúlveda J. Metodología de la Encuesta Nacional Seroepidemiológica, México. Salud Publica Mex 1992;34:I24-I35. 44. Galván-Portillo MV,Wolff MS, Torres-Sánchez LE, López-Cervantes M, López-Carrillo L. Assessing phytochemical intake in a group of Mexican women. Salud Publica Mex 2007;49:126-131.

45. López-Carrillo L, López-Cervantes M,Ward HM, Bravo-Alvarado J y Ramírez-Alvarado A. Nutrient and gastric cancer in México. Int J Cancer 1999;83:60I-605.
46. Selhub J, Jacques PF, Dallal G, Choumenkovitch S, Rogers G. The use of blood concentrations of vitamins and their respective functional indicators to define folate and vitamin $B_{12}$ status. Food Nutr Bull 2008;29:S67-S73. 47. Willet W, Howe G \& Kushi L. Adjustment for total energy intake in epidemiologic studies. Am J Clin Nutr 1997; 65: 1220S-1228S.

48. Willet W and Lenart E. Reproducibility and Validity of Food Frequency Questionnaires. En: Nutritional Epidemiology. 2a edición, Oxford: Oxford University Press, 1998:101-147.

49. Henriquez-Sanchez P, Sanchez-Villegas A, Doreste-Alonso J, OrtizAndrellicchi A, Pfrimer K, Serra-Majem L. Dietary assessment meyhods for micronutrient intake: a systematic review on vitamins. Brit J Nut 2009; 102:S10S37.

50. Baric I,Atalic Z, Keser I, Cecic I \& Sucic M. Validation of the folate food frequency questionnaire with serum and erythrocyte folate and plasma homocysteine. Int J Food Sci Nutr 2009;60:10-18.

5I. Cade JE, Burley VJ,Warm DL, Thompson RL, Margetts BM. Foodfrequency questionnaires: a review of their design, validation and utilization. Nut Res Rev 2004;17:5-22.

52. Rivera-Dommarco J, Shamah-Levy T,Villalpando-Hernández S, González-de Cossío T, Hernández-Prado B, Sepúlveda J. Encuesta Nacional de Nutrición 1999. Estado nutricio de niños y mujeres en México. Cuernavaca, México: Instituto Nacional de Salud Pública, 200I.

53. Lyle BJ, Mares-Perlman JA, Klein BE, Klein R, Greger JL. Supplement users differ from nonusers in demographic, lifestyle, dietary and health characteristics.J Nutr 1998; 128:2355-2362.

54. Consejo Nacional contra las Adicciones-Instituto de Psiquiatría Ramón de la Fuente-Instituto Nacional de Salud Pública-Fundación Gonzalo Río Arronte IAP. Encuesta Nacional de Adicciones 2008. SSA-INEGI, Encuesta Nacional de Adicciones 2002 [monografía en internet]. México: Conadic, 2002. Disponible en: http://www.conadic.gob.mx (consultado: 8 marzo 200I). 